\title{
Te SHALLOW DONOR SOLUBILITY MECHANISM IN GaAs
}

\author{
T. SluPiński, E. ZiElińska-Rohozińska and T. HaRasimowicz
}

Inst. of Experimental Physics, Warsaw University, Hoża 69, 00-681 Warsaw, Poland

\begin{abstract}
Results of thermal annealing of extremely highly doped GaAs:Te on room temperature Hall electron concentration and diffuse X-ray scattering are briefly reported. Reversible decrease/increase in electron concentration vs. temperature of annealing perfectly coincides with a strong increase/decrease in diffuse X-ray scattering intensity. An analysis of X-ray results indicates an arising of correlations in impurity positions in crystal lattice points in GaAs:Te solid solution for very high doping level. We give a sketch of a new formal model of tight bond creation between impurity atoms, which can consistently describe our results. The model is free from difficulties in describing annealing results encountered by a widely spread model of charge compensation by native acceptors.
\end{abstract}

PACS numbers: $64.75 .+\mathrm{g}, 72.80$.Ey

It is known [1-3] that a maximum free electron concentration technologically available in GaAs doped with Te (and all other donor elements [4]) is lower than a total concentration of donor impurity atoms in a crystal. A charge compensation mechanism was proposed in $n$-GaAs with some native defects $\mathrm{V}_{\mathrm{Ga}}^{-3}$ [4], or $\left[\mathrm{Te}_{\mathrm{As}} \mathrm{V}_{\mathrm{Ga}}\right]^{-1}$ [2] as acceptors. This mechanism describes well the saturation of free electron concentration as a function of dopant concentration $[2,4]$, but it predicts [5] an opposite direction of free electron concentration changes $n(T)$ caused by annealing at high temperature $T$, than experimentally measured [6].

In this paper we present a sketch of arguments for an alternative model capable to explain the maximum free electron concentration. We show that if we formally suppose a certain type of the tight bond interaction between close donor impurity atoms, controlled by the Fermi level, we can consistently describe both free electron concentration limit and annealing results. We suppose that in very highly doped GaAs:Te one deals with a finite solubility of inpurity as isolated dopant atoms (which are thus shallow donors) and impurity pairs/molecules/small clusters occur. The total solubility of dopant is much higher than the solubility in shallow donor form.

To test this hypothesis we used thermal annealing, which introduces a perturbation into the state of equilibrium of defects. We measured the changes of the Hall electron concentration and diffuse X-ray scattering [7-9] as a function of annealing. 
The samples investigated were from melt-grown bulk crystals of GaAs doped with Te at several levels $n=(1-15) \times 10^{18} \mathrm{~cm}^{-3}$ and double-doped with Te and Ge $n=(1-3) \times 10^{18} \mathrm{~cm}^{-3}$. The thermal treatments were done at $T=600-1200^{\circ} \mathrm{C}$ in such a way that samples were brought to an equilibrium at high temperature $T$ (annealing time for each temperature was long enough [6]) and very fast cooled down (quenched, cooling rate about $100^{\circ} \mathrm{C} / \mathrm{s}$ ) by throwing the ampoules with samples in water. The electron concentration after annealing was measured by the Hall effect in the van der Pauw configuration at room temperature. Advanced X-ray measurements - reciprocal space mapping, were taken using a high resolution Philips Materials Research Diffractometer in triple axis geometry [10] ( $\mathrm{Cu} K_{\alpha_{1}}$ radiation, Bartels type $\mathrm{Ge}$ monochromator, analyser, independent $\omega$ and $\omega / 2 \theta$ scans).

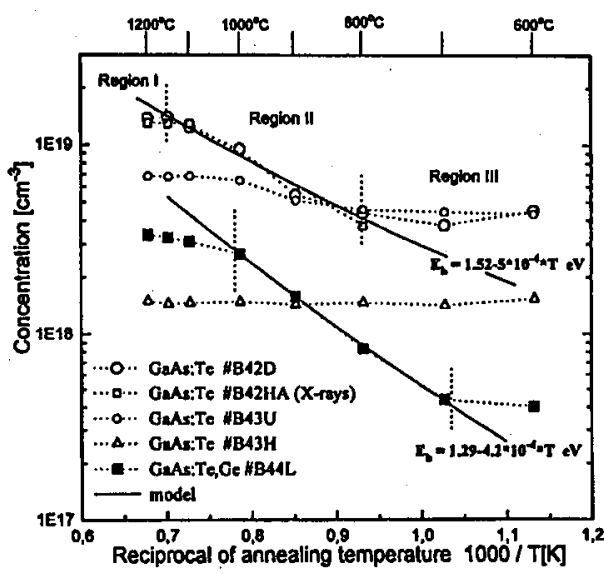

Fig. 1. Results of RT Hall electron concentration of annealed samples. Points measured for the same sample are connected with a dotted line. Open points are for single doped GaAs:Te, full points are for double doped GaAs:Te,Ge. Vertical dotied lines indicate characteristic regions of behaviour for samples GaAs:Te \#B42D and GaAs:Te,Ge \#B44L. Solid lines represent a fit in region II according to the model proposed.

Figure 1 presents the free electron concentration $n$ for a set of samples as a function of annealing temperature. For a very high doping level in GaAs:Te (sample \#B42D and \#B42HA) a strong decrease in $n$ after low temperature annealing (e.g. $800^{\circ} \mathrm{C}$ ) was measured. This decrease was fully reversible, subsequent annealing at high temperature (e.g. $1100^{\circ} \mathrm{C}$ ) restored high value of $n$. In a large number of annealing cycles done in this work the changes observed were always similar, $n$ could be switched to a low or high value depending on annealing temperature, undoubtedly indicating that reversible electrical deactivation is a property of bulk highly doped GaAs:Te itself.

The equilibrium value of the free electron concentration established for a given annealing temperature $T$ depends on a doping level and temperature. For 
the low doping level in GaAs:Te - sample \#B43H, annealing does not alter the value of $n$. For the high doping level - samples \#B42D, \#B42HA, \#B43U, three distinct regions of annealing behaviour vs. temperature exist (signed I, II and III for sample \#B42D). Above the certain threshold temperature - region I, no further changes were observed. The value of $n$ in region I was well consistent with technological doping level. This indicates that all donor impurities were electrically active in region I. Below the threshold temperature - region II, the annealing reduced $n$. This reduction means that a certain localised electronic level (we call it $E_{\mathrm{b}}$ ), whatever its nature is - we discuss it later, starts to capture electrons from the conduction band. Kinetics of this capture is relatively slow [6], e.g. an equilibrium at $800^{\circ} \mathrm{C}$ is attained after more than 100 hours of annealing, but at $1150^{\circ} \mathrm{C}$ after a few minutes only.

The equilibrium value of $n$ in region II for a given sample depends on temperature $T$ of annealing, the higher temperature $T$, the higher $n$. For lower doping level - sample \#B43U, the capture starts at lower temperature. These observations suggest that the relative position of the Fermi level $E_{\mathrm{F}}$ and $E_{\mathrm{b}}$ level depending on temperature, is the driving force for the capture. For higher temperature, when $E_{\mathrm{F}}$ should lie below $E_{\mathrm{b}}$, electrons captured are released from $E_{\mathrm{b}}$. At lower $T$, which should raise $E_{\mathrm{F}}$ upper relative to $E_{\mathrm{b}}$, the preference to localise electrons on $E_{\mathrm{b}}$ level exists.

Another observation is that below value of $n=2-4 \times 10^{18} \mathrm{~cm}^{-3}$ no further changes of $n$ occur in low annealingtemperatures - region III.

An identical character of annealing results (reversibility, three regions), but in a much lower free electron concentration range exists for crystals with two donor types, i.e. GaAs:Te,Ge. This suggests the same mechanism of changes caused by annealing. This mechanism must depend on chemical element of donor impurity, contrary to assumptions in the model of compensation by native acceptors [4].

Some suggestions about the structural defect introducing the $E_{\mathrm{b}}$ level can be drawn from X-ray studies. We consider diffuse X-ray scattering (DXS), i.e. the scattering accompanying Bragg diffraction, which is sensitive to all kinds of imperfections in a crystal [7-9], e.g. a state of order in a solid solution (pair correlation function [7-9], etc.), static deformations of lattice, phonons, etc. The intensity of DXS is usually much lower than the Bragg diffracted one. Specific features of DXS allow one to recognise the origin of imperfections, but it is very difficult to draw conclusions on details of defects $[8,9]$ due to the averaged and additive character of DXS from all defects.

Figure 2 presents an example of X-ray scattering intensity distribution (reciprocal space maps) in the vicinity of reciprocal lattice point (RLP) $h k l=004$, carried out for GaAs:Te sample \#B42HA from Fig. 1. The vector $q$ defines the deviation of the diffraction vector $\Delta k$ from the exact RLP: $q=\Delta k-h_{004}$, where $h_{004}$ is the (004) reciprocal lattice vector. Logarithmic isointensity contours as a function of $\boldsymbol{q}=\left[q_{x}, q_{z}\right]$ are shown. Diffuse X-ray scattering is observed as an excess intensity off 004 RLP (off the origin in Fig. 2). As seen, the annealing at $T=800^{\circ} \mathrm{C}$ caused a decrease in $n$ (Fig. 1) and an increase in DXS intensity - Fig. 2a. The subsequent annealing at $T=1150^{\circ} \mathrm{C}$ caused an increase in $n$ and about hundred times reduction of DXS intensity - Fig. 2b. Moreover, for low-doped GaAs:Te 

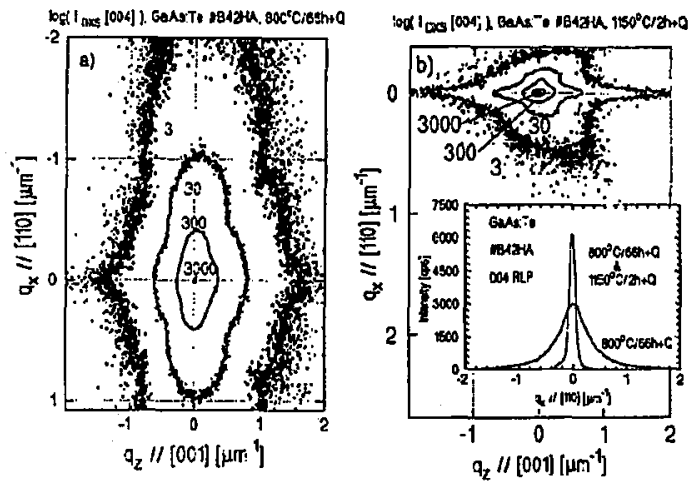

Fig. 2. Isointensity contours (logarithmic scale) of X-ray scattering around 004 RLP for GaAs:Te, (001)-oriented sample \#B42HA: (a) equilibrated at $T=800^{\circ} \mathrm{C}$, (b) subsequently equilibrated at $T=1150^{\circ} \mathrm{C}$. The $q_{x}$ and $q_{z}$ axis are parallel to $\langle 110\rangle$ and $\langle 001)$ directions of reciprocal lattice ( $q_{x}-\omega$ scan, $q_{z}-\omega / 2 \theta$ scan). An inset shows intensity sections along $q_{x}$ direction. Strong DXS observed after lowering of free electron concentration (part (a)) was about 100 times reduced (part (b)) after increasing electron concentration by high temperature treatment.

samples $\left(n<2 \times 10^{18} \mathrm{~cm}^{-3}\right)$, where no electrical changes occurred, low DXS was observed and annealing did not caused any significant changes. These strong coincidences indicate that a dominant part of DXS comes from structural defects created/disappeared during annealing. A close relation between an origin of measured DXS and the free electrons reduction mechanism has been established.

From very characteristic features of the isointensity contours observed, of its dependence on $h k l$ and vector $q$ we can infer, following $\S 19$ of Ref. [7], $\S 14$ of Ref. [8] and Ref. [9], that the changes of DXS are caused by an alteration of the state of order $[7-9,11]$ in the GaAs:Te solid solution, that is an occurrence of correlations of impurity atoms positions. The scattering close to RLP observed by us is due to the spatial fluctuations of impurity-impurity correlation function. Nonhomogeneity of this type in larger than the atomic scale (e.g. $1 \mu \mathrm{m})$ is a known property of solutions with arising correlations and is called the local order [7-9]. It means that a creation of one pair makes favourable the creation of another one in the vicinity.

This interpretation of DXS in GaAs:Te is different than the one known from GaAs literature, where defect-defect correlations are neglected. Such simplification is not proper for the solid solution close to the solubility limit [7-9] considered here. Due to a strong coincidence between electrical and X-ray results we can propose a formal model of impurity atoms interaction and pairing, capable to describe both electrical changes, its kinetics and an origin of impurity-impurity correlations. The $E_{\mathrm{b}}$ level, concluded from electrical measurements, is created only if two impurity atoms become close to each other. Thus the $E_{\mathrm{b}}$ level could be a certain type of the bonding state between the impurity atoms in their localised electron state $D^{*}$. The impurity atoms interaction is a balance of $D^{*}-D^{*}$ attraction 
and screened Coulomb repulsion - this allows one to substantiate region III in Fig. 1. Diffusion of donor atoms at elevated temperatures determines the kinetics of electron capture. For such a model we can easily fit the behaviour in Fig. 1 for region II, where the electron capture occurs, taking position of $E_{\mathrm{b}}$ vs. temperature as a fitting parameter. For a high temperature band structure of GaAs [12] (the valence band and conduction $\Gamma$ and $L$ bands were taken into calculations) we have got the best fit (the solid line in Fig. 1) for $E_{\mathrm{b}}[\mathrm{eV}]=1.52-5 \times 10^{-4} \mathrm{~T}[\mathrm{~K}]$ for Te doping and $E_{\mathrm{b}}[\mathrm{eV}]=1.29-4.2 \times 10^{-4} \mathrm{~T}[\mathrm{~K}]$ for $\mathrm{Te}+\mathrm{Ge}$ doping, relative to the valence band.

The details of this work will be presented elsewhere, here our purpose was to briefly describe the basic new ideas.

In conclusion, we demonstrate for the first time that the changes of free electron concentration vs. annealing in GaAs:Te are accompanied by the arising of correlations of impurity positions in a solid solution, e.g. impurity pairs. Supposing formally a certain type of the tight bond interaction between close impurity atoms we can satisfactorily describe the results of both annealing and the saturation of free electron concentration. Proposition of covalently bonded impurity atoms was put forward [6] to describe annealing many years ago and recently [13] to describe electrical deactivation and local vibrational mode (LVM) and Raman spectroscopy in $\delta$-doped GaAs:Si.

T.S. thanks Prof. M. Grynberg, Prof. M. Baj, Prof. J.A. Kozubowski and Prof. J. Baranowski for valuable discussions. The work was supported by the Committee for Scientific Research within grants 3P407-031-07 and 7T08A-044-08. MRD diffractometer was granted by the Foundation for Polish Science within SEZAM Program.

\section{References}

[1] H.C. Casey, M.B. Panish, K.B. Wolfstirn, J. Phys. Chem. Solids 32, 571 (1971).

[2] For a revue: D.T.J. Hurle, J. Phys. Chem. Solids 40, 627 (1979).

[3] M.C. Wu, Y.K. Su, K.Y. Cheng, C.Y. Chang, Solid State Electron. 31, 251 (1988).

[4] W. Walukiewicz, Appl. Phys. Lett. 54, 2094 (1989).

[5] W. Walukiewicz, Phys. Rev. B 39, 8776 (1989).

[6] C.S. Fuller, K.B. Wolfstirn, J. Appl. Phys. 34, 2287 (1963).

[7] M.A. Krivoglaz, Theory of X-Ray and Thermal Neutrons Scattering by Real Crystals, Plenum, New York 1969.

[8] M.A. Krivoglaz, Diffuse Scattering of X-rays and Neutrons by Fluctuations in Crystals, Springer-Verlag, Berlin 1996.

[9] D. de Fontaine; in: Solid State Physics, Eds. H. Ehrenreich, F. Seitz, D. Turnbull, Vol. 34, Academic Press, New York 1979, p. 74.

[10] P.F. Fewster, J. Appl. Crystallogr. 22, 64 (1989).

[11] Ch. Barrett, T.B Massalski, Structure of Metals, Pergamon Press, Oxford 1980, Ch. 11.

[12] J.S. Blakemore, J. Appl. Phys. 53, R123 (1982).

[13] J. Wagner, R.C. Newman, C. Roberts, J. Appl. Phys. 78, 2431 (1995). 